\title{
Acumulación de forraje de Lotus corniculatus L., en función a diferentes
} estrategias de cosecha

Perpetuo Álvarez Vázquez a

Juan de Dios Guerrero Rodríguez ${ }^{\text {b* }}$

Gabino García De Los Santos ${ }^{c}$

María Esther Ortega Cerrilla ${ }^{\mathrm{d}}$

Sergio Iban Mendoza Pedroza ${ }^{\mathrm{d}}$

Santiago Joaquín Cancino ${ }^{\mathrm{e}}$

a Universidad Autónoma Agraria Antonio Narro. Departamento de Recursos Naturales Renovables. México.

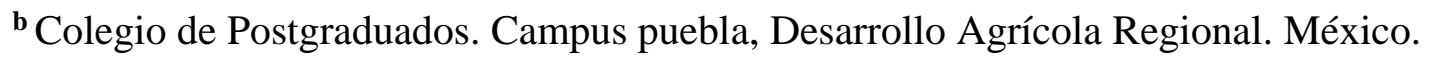

c Colegio de Postgraduados. Campus Montecillo, Recursos Genéticos y Productividad Producción de Semillas. México.

${ }^{\mathrm{d}}$ Colegio de Postgraduados. Campus Montecillo, Recursos Genéticos y ProductividadGanadería. Estado de México, México.

e Universidad Autónoma de Tamaulipas. Facultad de Ingeniería y Ciencias. México.

*Autor de correspondencia: grjuan2000mx@yahoo.com

\section{Resumen:}

El objetivo del estudio fue determinar la mejor estrategia de cosecha en Lotus corniculatus, dependiente de diferentes porcentajes de luz interceptada (LI) por el dosel y una denominada corte fijo (CF) definido estacionalmente, en dos periodos de producción. Los tratamientos (LI y CF) se distribuyeron en un diseño de bloques al azar, con tres repeticiones. El 
rendimiento de forraje del CF fue $27 \%$ menor al $95 \%$ de LI en el primer periodo $(19,915 v s$ $28,417 \mathrm{~kg} \mathrm{MS} \mathrm{ha}^{-1}$ ), y $29 \%$ al promedio de los porcentajes de LI en el segundo periodo $\left(19,100\right.$ vs 26,952 $\left.\mathrm{kg} \mathrm{MS} \mathrm{ha}^{-1}\right)$. El rendimiento promedio estacional fue mayor en primavera $\left(9,447 \mathrm{~kg} \mathrm{MS} \mathrm{ha}^{-1}\right)$, comparado con otoño $\left(3,120 \mathrm{~kg} \mathrm{MS} \mathrm{ha}^{-1}\right)$, en ambos periodos. La hoja fue el componente que mayor aporte tuvo (56\%), principalmente en primavera con $95 \%$ de LI. Con 90, 95 y $100 \%$ de LI se registraron las alturas mayores $(21.5 \mathrm{~cm})$ y con un CF las alturas menores $(17 \mathrm{~cm})$. Entre estaciones, las alturas mayores $(24 \mathrm{~cm})$ se presentaron en primavera y las menores en invierno $(17 \mathrm{~cm})$. En ambos periodos, se presentó la relación promedio hoja:tallo mayor en el CF (2.3), seguido del 90, 95 y $100 \%$ de LI. El comportamiento mejor de Lotus corniculatus genotipo 255301, se presentó cuando éste fue cosechado usando los porcentajes de luz interceptada como indicador; sin embargo, la mayor cantidad de hoja se produjo con $95 \%$ de luz interceptada.

Palabras clave: Lotus corniculatus L., Producción de forraje, Estrategia de cosecha, Luz interceptada.

Recibido: 15/06/2018

Aceptado: 23/10/2019

\section{Introducción}

Lotus corniculatus L., conocida comúnmente como trébol pata de pájaro, es la especie forrajera de mayor importancia de su género; registra alrededor de 200 especies entre anuales y perennes ${ }^{(1)}$, las cuales ocupan un $90 \%$ del área sembrada en el mundo ${ }^{(2)}$. Es comparada con la alfalfa (Medicago sativa L.) y el trébol blanco (Trifolium repens L.), ya que, su rendimiento y su calidad nutricional (entre 18.9 a $21.8 \%$ de proteína cruda, en base seca), es similar o superior a estas especies ${ }^{(3)}$. Contiene además, menos celulosa y más carbohidratos no estructurales ${ }^{(4)}$ y no produce meteorismo en rumiantes en pastoreo, debido a la presencia de taninos condensados ${ }^{(5)}$. Sin embargo, como en el resto de las especies forrajeras su productividad y persistencia están en función de la acumulación de forraje, y ambas características son determinadas por la estrategia de cosecha ${ }^{(6)}$, a la par de una eficiencia en ésta ${ }^{(7)}$.

Así mismo, el crecimiento de las plantas y las prácticas de manejo, son variables que interaccionan con el suelo y clima ${ }^{(8)}$, por lo tanto, a medida que avanza el crecimiento de la planta se propicia la competencia entre ellas; principalmente, en los periodos de rebrote, donde se reduce la cantidad y calidad luminosa en la pradera ${ }^{(9)}$. En otros estudios mencionan que, cuando en la pradera se alcanza un valor de $95 \%$ de luz interceptada, se llega a un punto 
óptimo de cosecha, donde se obtiene la mejor productividad ${ }^{(10)}$. En consecuencia, un buen manejo de la cantidad de luz interceptada por la pradera, asegura una mejor productividad de la misma ${ }^{(11)}$. En leguminosas de clima templado se han encontrado correlaciones altas entre la acumulación de forraje y la cantidad de luz interceptada ${ }^{(12)}$, no obstante, en L. corniculatus, es poco lo que se ha generado en investigación a este respecto. Por lo tanto, el objetivo de este trabajo fue determinar la mejor estrategia de cosecha de Lotus corniculatus, genotipo 255301, sometido a cuatro intervalos de cosecha; tres dependientes del porcentaje de intercepción de luz y un corte fijo definido estacionalmente.

\section{Material y métodos}

Se realizaron dos experimentos en condiciones de campo en el periodo de otoño-verano 2014-2015 (POV1) y otoño-verano 2015-2016 (POV2), en el Colegio de Postgraduados, Texcoco, México ( $19^{0} 29^{\prime} \mathrm{N}, 98^{0} 54^{\prime}$ O y 2,250 msnm). La textura del suelo es franco arenosa, ligeramente alcalina, con un $\mathrm{pH}$ de $7.8^{(13)}$. El clima del lugar es templado subhúmedo con régimen de lluvias en verano, precipitación y temperatura media anual de $645 \mathrm{~mm}$ y $15{ }^{0} \mathrm{C}$, respectivamente ${ }^{(14)}$.

Durante el estudio, los datos de temperatura del aire (mínima y máxima) y precipitación durante se obtuvieron de la estación meteorológica de la Universidad Autónoma Chapingo (Figura 1), ubicada a $2 \mathrm{~km}$ de distancia del área experimental. La precipitación acumulada del POV1 fue de 1,043 mm y del POV2 de $877 \mathrm{~mm}$. En ambos periodos, las máximas temperaturas se presentaron en primavera-verano.

Figura 1: Temperatura media mensual máxima, mínima y precipitación acumulada mensual

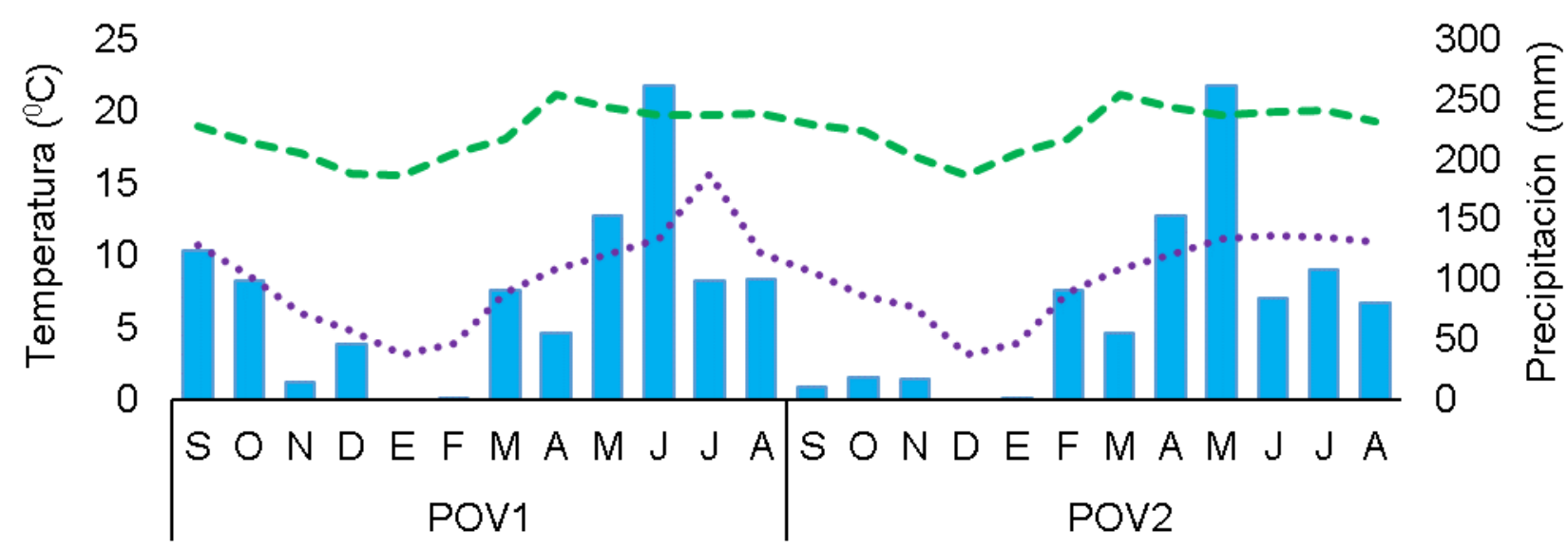

PP $\quad$ - - Temp. Máxima …..Temp. Mínima

POV1=Periodo de otoño 2014-verano 2015; POV2 = Periodo de otoño 2015-verano 2016. Datos de la estación meteorológica Universidad Autónoma Chapingo. 
Se evaluó una pradera de Lotus corniculatus L., genotipo 255301, establecida mediante trasplante en marzo de 2014, a una distancia entre plantas de $33 \mathrm{~cm}$. Las plantas se obtuvieron de material reproducido en invernadero. No se aplicó ningún tipo de fertilizante y en las estaciones de poca o nula precipitación, se proporcionaron riegos a capacidad de campo cada dos semanas. Al inicio del estudio (5 de septiembre de 2014), se realizó un corte manual a 7 $\mathrm{cm}$ sobre el nivel del suelo, para uniformización de la altura del forraje. La unidad experimental consistió en parcelas de $4 \mathrm{~m}^{2}$. Los tratamientos consistieron en cuatro estrategias de cosecha manual; tres intervalos de corte cuando la pradera alcanzó porcentajes de luz interceptada de 90, 95 y $100 \%$ y uno denominado corte fijo definido por estación del año; otoño: 35 , invierno: 42 , y primavera -verano: 28 días entre corte, a una altura de forraje residual de $7 \mathrm{~cm}$ en todos los tratamientos ${ }^{(3)}$. Los porcentajes de luz interceptada por la pradera se monitorearon previos al corte, tomando seis lecturas en cada parcela a las $1200 \mathrm{~h}$, utilizando un ceptómetro modelo LP-80 (Decagon Devices, EE.UU). Los cuatro tratamientos se asignaron aleatoriamente en cuatro parcelas de $4 \mathrm{~m}^{2}$ en un diseño de bloques completamente al azar con tres repeticiones, cuatro parcelas por bloque, generando 12 parcelas experimentales.

El rendimiento de forraje ( $\mathrm{kg} \mathrm{MS} \mathrm{ha}{ }^{-1}$ ) se determinó con la biomasa cosechada en dos cuadrantes fijos de $0.25 \mathrm{~m}^{2}$ por repetición, establecidos al inicio del experimento. El material cosechado se depositó en bolsas etiquetadas y se deshidrató a $60{ }^{\circ} \mathrm{C}$ hasta peso constante en una estufa de aire forzado (Felisa, Mod. FE-243A). Del forraje cosechado se cuantificó la composición botánica y morfológica (CBM), tomando una sub-muestra de aproximadamente del $10 \%$, la cual se separó en hoja, tallo, material muerto (material senescente) y maleza y se calculó el aporte al rendimiento en $\mathrm{kg} \mathrm{MS} \mathrm{ha}^{-1}$. Los datos obtenidos de hoja y tallo, de la CBM, se utilizaron para determinar la relación hoja:tallo, al dividir el peso de la hoja entre el tallo. Por otra parte, para estimar la altura de planta promedio, un día antes del corte, se tomaron al azar 12 lecturas por repetición, con una regla graduada a $50 \mathrm{~cm}$ y se promediaron los valores de los cortes pertenecientes a cada estación. De estos, se calculó la equivalencia en rendimiento de forraje por centímetro de altura de planta, dividiendo el rendimiento entre la altura entre el número de cortes ${ }^{(15)}$.

Para investigar el efecto de los tratamientos (estrategia de cosecha) sobre las variables de respuesta, los datos se agruparon de forma estacional y por periodo de estudio, esto para facilitar su análisis y discusión. Se analizaron conforme a un diseño experimental de bloques al azar con cuatro tratamientos y tres repeticiones, mediante el procedimiento PROC GLM del paquete estadístico $S A S^{(16)}$. La comparación de medias se realizó por medio de la prueba Tukey $(P \leq 0.05)$. 


\section{Resultados y discusión}

\section{Rendimiento de forraje}

Los mayores rendimientos promedios estacionales se presentaron en primavera $(9,447 \mathrm{~kg}$ MS ha ${ }^{-1}$ ), sin ser diferentes a verano del POV1, y los menores en otoño (3,120 kg), siendo similar a invierno del mismo periodo (Cuadro 1). Este comportamiento tuvo relación directa con las temperaturas óptimas $\left(22{ }^{0} \mathrm{C}\right)$ para el crecimiento de Lotus corniculatus ${ }^{(3)}$. Las temperaturas favorables se presentaron en primavera (Figura 1), lo cual benefició el crecimiento y producción de la especie. Esto concuerda con lo reportado en cinco poblaciones de L. corniculatus, al utilizar un intervalo de pastoreo severo y uno intenso de 20 y 40 días, respectivamente ${ }^{(17)}$. Por lo tanto, los cambios estacionales en el rendimiento de una especie forrajera, pueden ser atribuidos a las condiciones ambientales presentes en cada estación del año ${ }^{(18)}$. Al respecto, se han encontrado distribuciones estacionales en la producción de forraje de $L$. corniculatus de 32, 30, 23 y $15 \%$ para primavera, verano, invierno y otoño, respectivamente, concentrándose un $62 \%$ en primavera-verano ${ }^{(19)}$. También, se han registrado rendimientos promedios de 7,700 $\mathrm{kg} \mathrm{MS} \mathrm{ha}^{-1}$, en un intervalo de corte de 45 días, en Texcoco, Edo. de México, influenciados por el clima, manejo y hábito de crecimiento del genotipo ${ }^{(3)}$.

Cuadro 1: Rendimiento de forraje $\left(\mathrm{kg} \mathrm{MS} \mathrm{h}^{-1}\right)$ de L. corniculatus, genotipo 255301, en función del porcentaje de luz interceptada (LI) y un corte fijo definido estacionalmente

\begin{tabular}{lllllll}
\hline LI $(\%)$ & Otoño & Invierno & Primavera & Verano & Acumulado & EEM \\
\cline { 2 - 7 } POV1 & & & & & & \\
90 & $4527 \mathrm{Ab}$ & $2736 \mathrm{Bb}$ & $10326 \mathrm{Aa}$ & $9746 \mathrm{Aa}$ & $27336 \mathrm{AB}$ & 1271 \\
95 & $4956 \mathrm{Ab}$ & $4422 \mathrm{Ab}$ & $10346 \mathrm{Aa}$ & $9942 \mathrm{Aa}$ & $28417 \mathrm{~A}$ & 481 \\
100 & $4235 \mathrm{Ab}$ & $2716 \mathrm{Bb}$ & $11002 \mathrm{Aa}$ & $9178 \mathrm{Aa}$ & $27132 \mathrm{AB}$ & 802 \\
Corte fijo & $3300 \mathrm{Ab}$ & $2431 \mathrm{Bb}$ & $8147 \mathrm{Aa}$ & $6851 \mathrm{Ba}$ & $20730 \mathrm{~B}$ & 529 \\
Promedio & $4255 \mathrm{~b}$ & $3076 \mathrm{~b}$ & $9956 \mathrm{a}$ & $8929 \mathrm{a}$ & 25904 & 564 \\
EEM & 683 & 522 & 1432 & 637 & 2461 & \\
POV2 & & & & & & \\
90 & $4749 \mathrm{Ac}$ & $3247 \mathrm{Ad}$ & $9953 \mathrm{Aa}$ & $8565 \mathrm{Ab}$ & $26515 \mathrm{~A}$ & 355 \\
95 & $4676 \mathrm{Ab}$ & $3835 \mathrm{Ab}$ & $9087 \mathrm{ABa}$ & $9515 \mathrm{Aa}$ & $27113 \mathrm{~A}$ & 505 \\
100 & $5501 \mathrm{Ac}$ & $3749 \mathrm{Ad}$ & $9732 \mathrm{Aa}$ & $8246 \mathrm{Ab}$ & $27227 \mathrm{~A}$ & 477 \\
Corte fijo & $4603 \mathrm{Ab}$ & $1826 \mathrm{Bc}$ & $6982 \mathrm{Ba}$ & $5689 \mathrm{Bb}$ & $19100 \mathrm{~B}$ & 402 \\
Promedio & $4882 \mathrm{c}$ & $3164 \mathrm{~d}$ & $8938 \mathrm{a}$ & $8004 \mathrm{~b}$ & 24989 & 306 \\
EEM & 628 & 329 & 940 & 668 & 2397 & \\
\hline
\end{tabular}

Corte fijo; otoño: 35, invierno: 42, y primavera -verano: 28 días entre corte. POV1= periodo de otoño 2014verano 2015; POV2= periodo de otoño 2015-verano 2016. EEM = error estándar de la media.

Promedios con letra mayúscula diferente en una columna y letra minúscula diferente en una hilera son diferentes $(P \leq 0.05)$. 
En ambos periodos de estudio, los tratamientos presentaron diferencias $(P \leq 0.05)$ en el forraje acumulado. El rendimiento menor correspondió al corte fijo con $27 \%$ menos respecto al $95 \%$ LI (28,417 vs 20,730 kg MS ha-1) en el POV1 y $29 \%$ respecto al promedio de los tres porcentajes de luz interceptada (26,952 vs 19,100 $\mathrm{kg} \mathrm{MS} \mathrm{ha}^{-1}$ ) en el POV2 (Cuadro 1). Lo anterior, puede estar relacionado con un intervalo de cosecha menor de 33 días, correspondiente al corte fijo, respecto al intervalo de cosecha promedio de 70 días de los tratamientos cosechados con base a las intercepciones luminosas (Figura 2). En el cultivar 202700 en Texcoco, México, se reportó una menor adaptación de la especie Lotus corniculatus a un corte definido por estación, respecto a, cortes dependientes del porcentaje de luz interceptada, ya que, rindió $29 \%$ menos forraje que el resto de los tratamientos ${ }^{(20)}$. En algunas especies forrajeras, los cortes frecuentes disminuyen su rendimiento y área foliar, e incrementan la presencia de especie indeseables ${ }^{(21)}$ y con ello una mayor competencia con la especie deseada y el agotamiento de sus reservas de carbohidratos ${ }^{(22)}$.

Figura 2: Intervalos de corte (promedios), de L. corniculatus, genotipo 255301

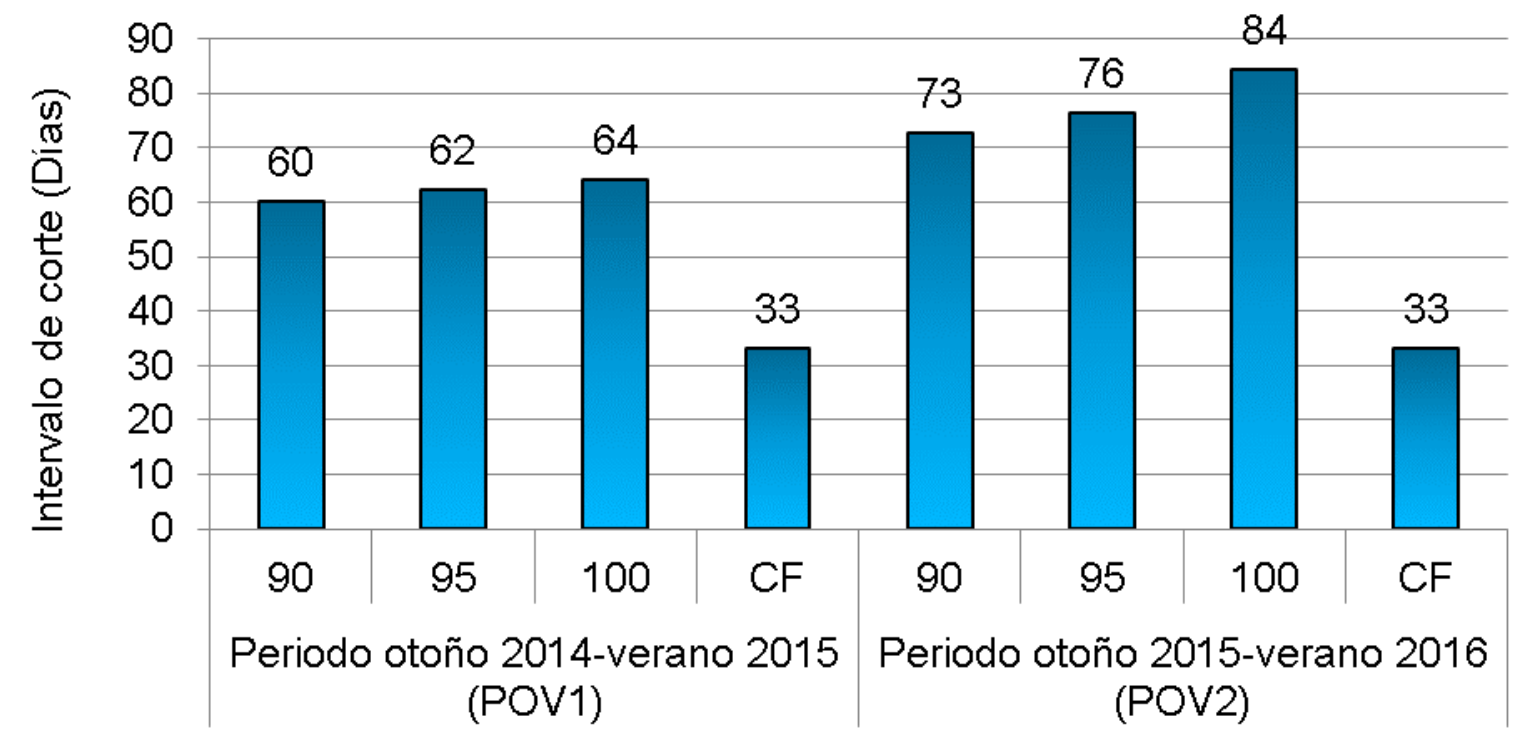

Tres cuando la pradera alcanzó porcentajes de luz interceptada de 90,95 y $100 \%$ y uno denominado corte fijo (CF) definido por estación; otoño: 35, invierno: 42, y primavera -verano: 28 días entre corte.

\section{Composición botánica y morfológica}

Dentro de los componentes morfológicos, la hoja fue la que más aportó al rendimiento con promedio de 14,273 $\mathrm{kg} \mathrm{MS} \mathrm{ha-1}$, que representó el $56 \%$, seguido por el tallo (30.5\%), material muerto $(8.5 \%$ ) y maleza $(4.5 \%)$. Entre tratamientos, con $95 \%$ de luz interceptada se presentó mayor cantidad de hoja con un promedio de 16,526 $\mathrm{kg} \mathrm{MS} \mathrm{ha}^{-1}$, siendo diferente $(P \leq 0.05)$ al resto de los tratamientos en el POV2. El corte fijo, presentó menor cantidad de hojas y de tallos con 12,276 y 4,710 $\mathrm{kg} \mathrm{MS} \mathrm{ha}^{-1}$, en ambos periodos, respectivamente. El 
material muerto y la maleza, no presentaron diferencias $(P \geq 0.05)$, en ambos periodos (Cuadro 2).

Cuadro 2: Rendimiento de forraje acumulado estacional ( $\mathrm{kg} \mathrm{MS} \mathrm{h}^{-1}$ ), por componente botánico y morfológico de L. corniculatus, genotipo 255301, en función del porcentaje de luz interceptada (LI) y un corte fijo definido estacionalmente

\begin{tabular}{|c|c|c|c|c|c|}
\hline LI (\%) & Hoja & Tallo & MM & Maleza & EEM \\
\hline \multicolumn{6}{|l|}{ POV1 } \\
\hline 90 & $13829 \mathrm{Aa}$ & $8426 \mathrm{Ab}$ & 3337 Ac & 459 Ad & 961 \\
\hline 95 & $15979 \mathrm{Aa}$ & $9450 \mathrm{Ab}$ & $2051 \mathrm{Ac}$ & $937 \mathrm{Ac}$ & 836 \\
\hline 100 & $14540 \mathrm{Aa}$ & $7964 \mathrm{ABb}$ & $4622 \mathrm{Ac}$ & $1291 \mathrm{Ac}$ & 754 \\
\hline Corte fijo & $12715 \mathrm{Aa}$ & $5364 \mathrm{Bb}$ & $2616 \mathrm{Ab}$ & $1329 \mathrm{Ab}$ & 1692 \\
\hline Promedio & $14266 \mathrm{a}$ & $7801 \mathrm{~b}$ & $3156 \mathrm{c}$ & $1004 \mathrm{~d}$ & 753 \\
\hline EEM & 1332 & 1048 & 971 & 662 & \\
\hline \multicolumn{6}{|l|}{ POV2 } \\
\hline 90 & $14315 \mathrm{ABa}$ & $7996 \mathrm{Bb}$ & 1608 Ac & $970 \mathrm{Ac}$ & 1022 \\
\hline 95 & $17074 \mathrm{Aa}$ & $9412 \mathrm{ABb}$ & $969 \mathrm{Ac}$ & $719 \mathrm{Ac}$ & 877 \\
\hline 100 & $13893 \mathrm{ABa}$ & $10403 \mathrm{Ab}$ & 2326 Ac & $605 \mathrm{Ad}$ & 533 \\
\hline Corte fijo & $11838 \mathrm{Ba}$ & $4056 \mathrm{Cb}$ & 1718 Ac & $2053 \mathrm{Abc}$ & 748 \\
\hline Promedio & $14280 \mathrm{a}$ & 7967 b & $1655 \mathrm{c}$ & $1087 \mathrm{c}$ & 565 \\
\hline EEM & 1102 & 757 & 570 & 750 & \\
\hline
\end{tabular}

Corte fijo; otoño: 35, invierno: 42, y primavera -verano: 28 días entre corte. POV1= periodo de otoño 2014verano 2015; POV2 = periodo de otoño 2015-verano 2016. EEM = error estándar de la media.

Promedios con letra mayúscula diferente en una columna y letra minúscula diferente en una hilera son diferentes $(P<0.05)$.

Una mayor producción de hoja y tallo con $95 \%$ LI, puede estar relacionada con un incremento en la edad de crecimiento del cultivo ${ }^{(23)}$. También, un aumento en la cantidad de hojas desde el corte fijo hasta un $95 \%$ LI, puede ser el resultado de una compensación en el incremento de la biomasa de los tallos, debido a un mayor periodo de crecimiento, que implica mayor tiempo produciendo fotosintatos ${ }^{(24)}$.

Estacionalmente, la hoja fue el componente que más aportó al rendimiento de forraje (Figura 3). El mayor y menor rendimiento promedio en ambos periodos se registró durante primavera e invierno con 5,141 y 1,580 $\mathrm{kg} \mathrm{MS} \mathrm{ha}^{-1}$, donde la estrategia de cosecha con $95 \%$ de luz interceptada y el corte fijo, presentaron los mayores y menores rendimientos de 5,852 y $1,163 \mathrm{~kg} \mathrm{MS} \mathrm{ha}^{-1}$, respectivamente. También en primavera e invierno se presentaron la mayor y menor cantidad de tallos con 3,347 y $772 \mathrm{~kg} \mathrm{MS} \mathrm{ha}^{-1}$. Estos resultados pueden ser consecuencia de las condiciones ambientales concurrentes en cada estación del año, presentes durante el estudio (Figura 1). Al respecto, se ha encontrado que los cambios en la producción de tallos están en función de las variaciones estacionales por la cantidad y calidad de luz, la 
precipitación y la temperatura, que incide sobre la pradera ${ }^{(18)}$. En cuanto al material muerto y la maleza, la mayor producción correspondió a verano con 873 y $268 \mathrm{~kg} \mathrm{MS} \mathrm{ha}^{-1}$, sin ser diferente $(P \geq 0.05)$ a primavera del primer periodo, mientras la menor a otoño con 237 y 133 $\mathrm{kg} \mathrm{MS} \mathrm{ha}{ }^{-1}$, respectivamente. Lo anterior, puede ser el resultado de un auto sombreado de la planta a nivel del área basal, debido a un mayor crecimiento de esta en las estaciones con condiciones favorables para su desarrollo ${ }^{(25)}$.

Figura 3: Composición botánica y morfológica de L. corniculatus, genotipo 255301, en función del porcentaje de luz interceptada y un corte fijo $(\mathrm{CF})$ definido por estación= otoño: 35 , invierno: 42 , y primavera -verano: 28 días entre corte

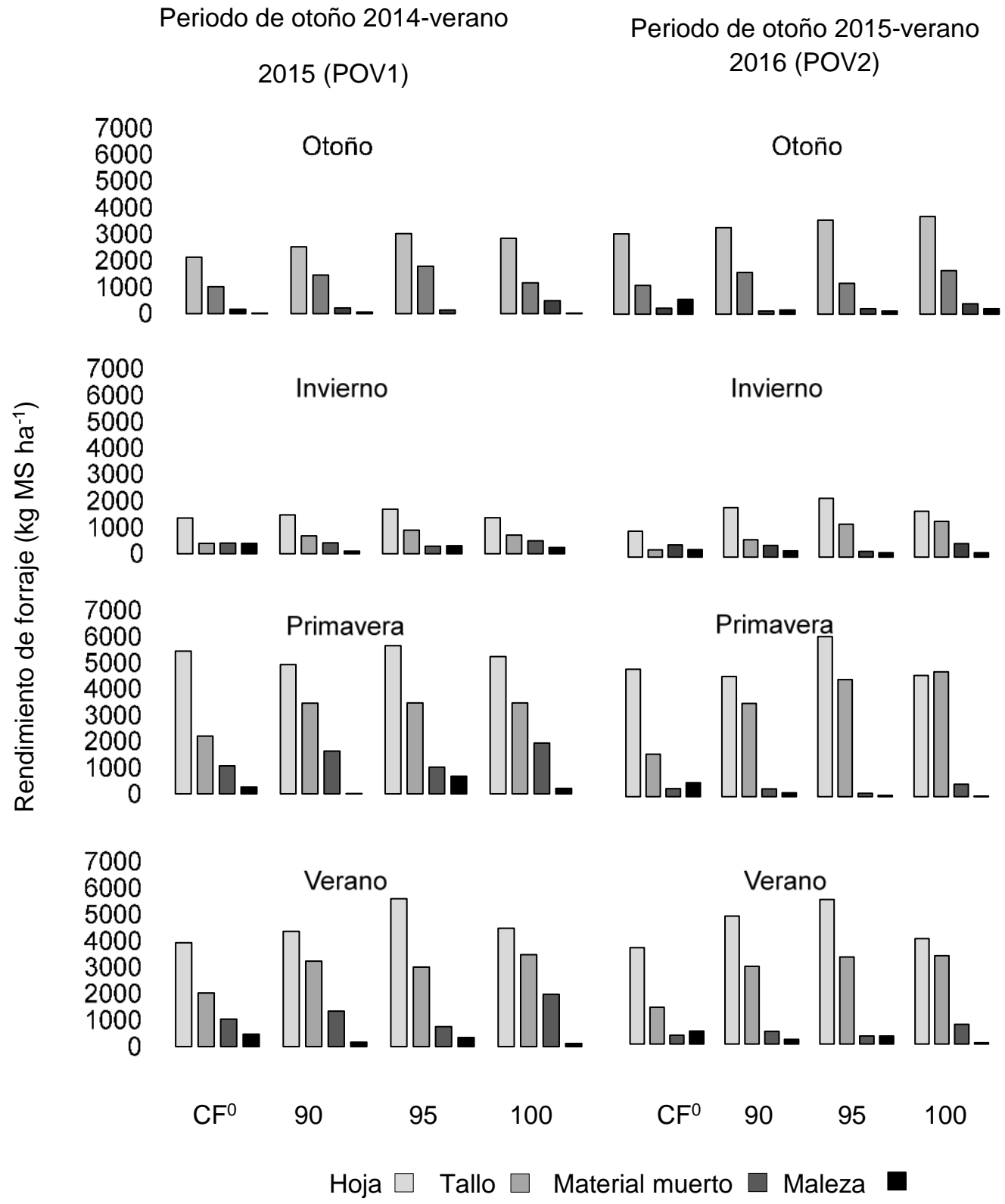




\section{Altura de planta}

Hubo diferencias entre tratamientos y estaciones $(P \leq 0.05)$. Las alturas mayores, se registraron en 90,95 y $100 \%$ de luz interceptada con $21.5 \mathrm{~cm}$ promedio, siendo diferentes $(P \geq 0.05)$ al $90 \%$ de IL en POV2, mientras que, las alturas menores se presentaron en el corte fijo con $17 \mathrm{~cm}$, en promedio de ambos periodos (Cuadro 3). Estos resultados están relacionados con la edad de la pradera, ya que, en parcelas cosechadas en función del porcentaje de luz interceptada, la planta tuvo más días de rebrote con 70 días respecto al corte fijo con 33 días, promedio de ambos periodos (Figura 2). En el corte fijo, la edad de la planta fue menor, con cortes más frecuentes, reflejándose en un rendimiento de forraje menor ${ }^{(17)}$. $\mathrm{Al}$ respecto, se ha señalado que a una altura mayor corresponde un rendimiento mayor de forraje ${ }^{(15)}$ y en el caso de Lotus corniculatus, el rendimiento y la altura, también se ha relacionado con los hábitos de crecimiento erectos y postrados ${ }^{(3)}$.

Cuadro 3: Altura de planta (cm) de L. corniculatus, genotipo 255301, en función del porcentaje de luz interceptada (LI) y un corte fijo definido estacionalmente

\begin{tabular}{lllllll}
\hline LI $(\%)$ & Otoño & Invierno & Primavera & Verano & Promedio & EEM \\
\cline { 2 - 5 } POV & $21 \mathrm{ABa}$ & $18 \mathrm{Ab}$ & $23 \mathrm{Aa}$ & $24 \mathrm{Aa}$ & $22 \mathrm{~A}$ & 0.9 \\
90 & $23 \mathrm{Aa}$ & $17 \mathrm{Aa}$ & $23 \mathrm{Aba}$ & $24 \mathrm{Aa}$ & $22 \mathrm{~A}$ & 2.4 \\
95 & $18 \mathrm{Bc}$ & $20 \mathrm{Abc}$ & $24 \mathrm{Aa}$ & $23 \mathrm{Aab}$ & $21 \mathrm{~A}$ & 1.6 \\
100 & $24 \mathrm{Aa}$ & $12 \mathrm{Bc}$ & $21 \mathrm{Bab}$ & $18 \mathrm{Bb}$ & $19 \mathrm{~B}$ & 1.5 \\
Corte fijo & $21 \mathrm{a}$ & $17 \mathrm{~b}$ & $23 \mathrm{a}$ & $22 \mathrm{a}$ & 21 & 1.5 \\
Promedio & 1.6 & 1.1 & 0.6 & 0.7 & 0.8 & \\
EEM & & & & & & \\
POV2 & $19 \mathrm{Ab}$ & $13 \mathrm{Bc}$ & $24 \mathrm{Ba}$ & $24 \mathrm{ABa}$ & $20 \mathrm{~B}$ & 1.0 \\
90 & $17 \mathrm{ABc}$ & $22 \mathrm{Ab}$ & $30 \mathrm{Aa}$ & $24 \mathrm{Ab}$ & $23 \mathrm{~A}$ & 1.1 \\
95 & $19 \mathrm{Ac}$ & $26 \mathrm{Ab}$ & $31 \mathrm{Aa}$ & $22 \mathrm{ABbc}$ & $25 \mathrm{~A}$ & 1.2 \\
100 & $15 \mathrm{Bb}$ & $8 \mathrm{Cc}$ & $16 \mathrm{Cb}$ & $22 \mathrm{Ba}$ & $15 \mathrm{C}$ & 0.8 \\
Corte fijo & $18 \mathrm{c}$ & $17 \mathrm{c}$ & $25 \mathrm{a}$ & $23 \mathrm{~b}$ & 21 & 0.4 \\
Promedio & 1.2 & 1.3 & 0.9 & 0.9 & 0.5 & \\
EEM & 1.2 &
\end{tabular}

Corte fijo = otoño: 35 , invierno: 42 , y primavera -verano: 28 días entre corte. POV1= periodo de otoño 2014verano 2015; POV2= periodo de otoño 2015-verano 2016. EEM = error estándar de la media.

Promedios con letra mayúscula diferente en una columna y letra minúscula diferente en una hilera son diferentes $(P \leq 0.05)$. 
Entre estaciones, en ambos periodos de estudio, la altura promedio mayor se registró en primavera con $24 \mathrm{~cm}$, siendo similar a verano y otoño $(P \geq 0.05)$ en el POV1, y la menor en invierno con $17 \mathrm{~cm}$, siendo similar a otoño $(P \geq 0.05)$, en el POV2. Las alturas mayores de la planta se encontraron en las estaciones con condiciones más óptimas de humedad y temperatura para su crecimiento y rendimiento de la especie (Figura 1). Estos resultados son similares a los reportados en el Estado de México, México; en 12 genotipos de $L$. corniculatus $^{(3)}$. También, se ha reportado que en condiciones adecuadas de fotoperiodo, temperatura y humedad, las plantas forrajeras aceleran su crecimiento y presentan cambio en la altura en las diferentes estaciones ${ }^{(26)}$, lo cual, tiene una relación directa con el rendimiento de forraje ${ }^{(12)}$, asociado con el hábito de crecimiento postrado del genotipo $255301^{(3)}$. Es así que, la altura de la pradera da una idea del forraje producido ${ }^{(8)}$. Por lo tanto, al calcular la equivalencia de los mayores rendimientos estacionales y de los tratamientos, por centímetro de altura de planta, se obtuvo que cada centímetro correspondió $167 \mathrm{~kg} \mathrm{MS} \mathrm{ha}^{-1}$, promedio de ambos periodos, para primavera y $144 \mathrm{~kg} \mathrm{MS} \mathrm{ha}^{-1}$, para el $95 \%$ LI (POV1) y $192 \mathrm{~kg}$, para el promedio de las intercepciones 90, 95 y $100 \%$ del POV2.

\section{Relación hoja:tallo}

La relación promedio mayor de hoja:tallo se presentó en el corte fijo $(P \leq 0.05)$, seguido de 90, 95 y $100 \%$ de luz interceptada, en ambos periodos (Cuadro 4). En el POV1, el corte fijo superó $36 \%$ al promedio de los tratamientos con 90, 95 y $100 \%$ de luz interceptada ( $2.8 \mathrm{vs}$ 1.8), mientras que en el POV2 este mismo tratamiento fue de $44 \%$ respecto al $100 \%$ de LI (3.2 vs 1.4). Esta relación hoja:tallo mayor en el corte fijo, fue el resultado de cosechas más frecuentes con 33 días promedio (Figura 2), lo cual no permitió que la especie fuese cosechada en su momento óptimo, sino, en la fase de crecimiento acelerado, donde se encuentra el mayor porcentaje de hojas jóvenes y poca cantidad de tallos ${ }^{(27)}$. Así mismo, posterior al corte, la calidad y cantidad de luz incidente al interior de la pradera, es alterada por el intervalo de corte que causa variaciones en la producción de hojas y tallos y por tanto, en la relación hoja:tallo ${ }^{(10)}$. 
Cuadro 4: Relación hoja:tallo de L. corniculatus, genotipo 255301, en función del porcentaje de luz interceptada y un corte fijo definido estacionalmente

\begin{tabular}{lllllll}
\hline LI $(\%)$ & Otoño & Invierno & Primavera & Verano & Promedio & EEM \\
\cline { 2 - 3 } & & & & & & \\
90 & $2.5 \mathrm{Aa}$ & $2.0 \mathrm{Bab}$ & $1.7 \mathrm{Bb}$ & $1.5 \mathrm{Ab}$ & $1.9 \mathrm{~B}$ & 0.2 \\
95 & $1.9 \mathrm{Aa}$ & $2.3 \mathrm{Ba}$ & $1.5 \mathrm{Ba}$ & $1.4 \mathrm{Aa}$ & $1.8 \mathrm{~B}$ & 0.3 \\
100 & $2.1 \mathrm{Aa}$ & $2.2 \mathrm{Ba}$ & $1.8 \mathrm{Ba}$ & $1.8 \mathrm{Aa}$ & $1.8 \mathrm{~B}$ & 0.2 \\
Corte fijo & $2.5 \mathrm{Ab}$ & $4.0 \mathrm{Aa}$ & $2.6 \mathrm{Ab}$ & $2.1 \mathrm{Ab}$ & $2.8 \mathrm{~A}$ & 0.4 \\
Promedio & $2.3 \mathrm{ab}$ & $2.6 \mathrm{a}$ & $1.9 \mathrm{~b}$ & $1.7 \mathrm{~b}$ & 2.1 & 0.2 \\
EEM & 0.2 & 0.3 & 0.2 & 0.4 & 0.2 & \\
POV2 & & & & & & \\
90 & $2.1 \mathrm{Ab}$ & $2.9 \mathrm{Ba}$ & $1.7 \mathrm{Bb}$ & $1.7 \mathrm{Bb}$ & $2.1 \mathrm{~B}$ & 0.1 \\
95 & $3.0 \mathrm{Aa}$ & $1.8 \mathrm{Cb}$ & $1.0 \mathrm{Cc}$ & $1.7 \mathrm{Bb}$ & $1.9 \mathrm{~B}$ & 0.1 \\
100 & $2.3 \mathrm{Aa}$ & $1.3 \mathrm{Cb}$ & $1.0 \mathrm{Cb}$ & $1.2 \mathrm{Cb}$ & $1.4 \mathrm{C}$ & 0.2 \\
Corte fijo & $2.9 \mathrm{Aa}$ & $3.7 \mathrm{Aa}$ & $3.4 \mathrm{Aa}$ & $2.7 \mathrm{Aa}$ & $3.2 \mathrm{~A}$ & 0.3 \\
Promedio & $2.6 \mathrm{a}$ & $2.4 \mathrm{a}$ & $1.8 \mathrm{~b}$ & $1.8 \mathrm{~b}$ & 2.2 & 0.1 \\
EEM & 0.3 & 0.2 & 0.2 & 0.1 & 0.1 & \\
\hline
\end{tabular}

Corte fijo= otoño: 35, invierno: 42 , y primavera -verano 28 días entre corte. POV1= periodo de otoño 2014verano 2015; POV2= periodo de otoño 2015-verano 2016. EEM= error estándar de la media.

Promedios con letra mayúscula diferente en una columna y letra minúscula diferente en una hilera son diferentes $(P \leq 0.05)$.

La relación hoja:tallo entre estaciones, fue mayor en invierno $(P \leq 0.05)$, con 2.6 en el POV1, y 2.5 promedio de otoño e invierno en el POV2. En algunas especies forrajeras se ha encontrado una relación hoja:tallo mayor en estaciones donde el crecimiento de las plantas es menor (otoño e invierno), debido a una mayor densidad de tallos, pero de menor peso ${ }^{(27)}$. En primavera y verano se registraron los menores valores, en ambos periodos $(P \leq 0.05)$, resultado de un peso individual de tallos mayor ${ }^{(26)}$, lo cual puede ser en respuesta a una translocación de asimilados mayor de las hojas hacia los tallos en estas $\operatorname{estaciones}^{(7)}$.

\section{Conclusiones e implicaciones}

Las estrategias de cosecha dependientes del porcentaje de luz interceptada tuvieron rendimientos de forraje y alturas de planta similares, marcando diferencia con el corte fijo definido estacionalmente, no obstante, esta última estrategia presentó mayor relación hoja:tallo, siendo la hoja, el componente morfológico que mayor aporte hizo al rendimiento de forraje en todos los tratamientos, principalmente cuando las parcelas fueron cosechadas con $95 \%$ de luz interceptada. 


\section{Literatura citada:}

1. Lagler JC. Lotus: Un género que no acaba en dos especies. Rev Forrajes y Granos 2003;(62):72-76.

2. Escaray FJ, Menendez AB, Gárriz A, Pieckenstain FL, Estrella MJ, Castagno LN, et al. Ecological and agronomic importance of the plant genus Lotus. Its application ingrassland sustainability and the amelioration of constrained and contaminated soils. Plant Sci 2012;(182):121-133.

3. García BDV, Guerrero RJD, García DSG. Lagunes RSA. Rendimiento y calidad de forraje de genotipos de Lotus corniculatus L., en el Estado de México. Nova Scientia 2014;7(13):170-189.

4. Grant FW. Lotus corniculatus. Sci Topics. 2009. http://www.scitopics.com/ Lotus_corniculatus.html. Accessed Sep 30, 2017.

5. MacAdam JW, Bruner J, Islam A, Shewmaker G. The benefits of tannin-containing forages. Plants, Soils and Climate, Utah State University AG/Forages 2013;03.

6. Da Silva NIM, Lima DSA, De Moura ZA, Gonçalves DAJ, De Jesus FD, Buranelo TFL, et al. Morphogenetic and structure characteristics of marandu grass subjected to grazing management strategies. Biolog Rhythm Res 2017;929-1016.

7. Giacomini AA, Da-Silva CS, Sarmento DLDO, Zeferino VC, Souza JS, Da-Trindade KJ, et al. Growth of marandu palisadegrass subjected to strategies of intermittent stocking. Sci Agric Piracicaba 2009;66(6):733-741.

8. Parsons A, Rowarth J, Thornley J, Newton P. Primary production of grassalands, herbage accumulation and use, and impacts of climate change. In: Lemaire G, et al. editors. Grassland productivity and ecosystems services. CABI, 2011:1-18.

9. Da Silva SC, Hernández GA. Manejo del pastoreo en praderas tropicales. En: Velazco ZME et al. editores. Los forrajes y su impacto en el trópico. 1ra. ed. Chiapas, México: Universidad Autónoma de Chiapas; 2010:63-95.

10. Montagner DB, Nascimento-Jr D, Vilela HH, Sousa MLB, Euclides BVP, Da-Silva CS, et al. Tillering dynamics in pastures of guinea grass subjected to grazing severities under intermittent stocking. Rev Bras Zootec 2012;41(3):544-549.

11. Difante GS, Nascimento-Jr D, Da-Silva SC, Bautista EVP, De-Moura ZA, Adese B. Dinâmica do perfilhamento do capim-marandu cultivado em duas alturas e três intervalos de corte. Rev Bras Zootec 2008;37(2):89-196. 
12. Rojas GAR, Hernández GA, Joaquín CS, Maldonado PMA, Mendoza PSI, Álvarez VP, et al. Comportamiento productivo de cinco variedades de alfalfa. Rev Mex Cienc Agríc 2016;7(8):1855-1866.

13. Delgado R, Escalante J, Díaz R, Trinidad SA, Morales E, Sosa E. Defoliación en maíz y su efecto sobre el rendimiento de frijol-maíz en asociación. Rev Mex Cienc Agríc 2014;5(6):1015-1027.

14. García E. Modificación al sistema de clasificación climática de Köppen. 4ta ed. Instituto de Geografía, Universidad Autónoma de México; 2004.

15. Hakl J, Hrevušova' Z, Hejcman M, Fuksa P. The use of a rising plate meter to evaluate lucerne (Medicago sativa $\mathrm{L}$.) height as an important agronomic trait enabling yield estimation. Grass Forage Sci 2012;67:589-596.

16. SAS Institute. SAS/STAT® 9.2. User Guide Release. Cary, NC: SAS Institute Inc. USA. 2009.

17. Scheffer BMS, Brustolin R, Dall AM. Performance of Lotus corniculatus L. genotypes submitted to cutting interval: subsidies to a breeding program. Rev Bras Zootec 2011;40(8):1645-1650.

18. Sbrissia AF, Da-Silva CS, Sarmento DOL, Molan LK, Andrade MF, Goncalves CA, et $a l$. Tillering dynamics in palisadegrass swards continuously stocked by cattle. Plant Eco 2010;(206):349-359.

19. Álvarez VP, Hernández GA, García DSG, Guerrero RJD, Mendoza PSI, Ortega CME, et al. Potencial forrajero de Lotus corniculatus L. con diferentes estrategias de manejo. Rev Agroproductividad 2018;11(5):24-28.

20. Álvarez VP, García DSG, Guerrero RJD, Mendoza PSI, Ortega CME, Hernández GA. Comportamiento productivo de Lotus corniculatus L. dependiente de la estrategia de cosecha. Rev Agrociencia 2018;52(8):1081-1093.

21. Mendoza PSI, Hernández GA, Pérez PJ, Quero CAR, Escalante EJAS, Zaragoza RJL, et $a l$. Respuesta productiva de la alfalfa a diferentes frecuencias de corte. Rev Mex Cienc Pecu 2010;1(3):287-296.

22. Teixeira EI, Derrick JM, Hamish BB, Fletcher LA. The dynamics of lucerne (Medicago sativa L.) yield components in response to defoliation frequency. Eur J Agron 2007;(26):394-400.

23. Gomide AMC, Chavesb SC, Ribeiroc KG, Sollenbergerd EL, Paciulloa SCD, Pereirae TP, et al. Structural traits of elephant grass (Pennisetum purpureum Schum.) genotypes under rotational stocking strategies. Afr J Range Forage Sci 2014:1-7. 
24. Pereira JC, Gomes FK, Oliveira DBLM, Lara ASM, Bernardes TF, Casagrande DR. Defoliation management affects morphogenetic and structural characteristics of mixed pastures of brachiaria grass and forage peanut. Afr J Range Forage Sci 2017;34(1):1319.

25. Baguet HA y Bavera GA. Fisiología de la planta pastoreada. Facultad de Agronomía y Veterinaria. Universidad Nacional del Río Cuarto. Provincia de Córdoba,Argentina2001.http://www.produccionovina.com.ar/produccioymanejopastra s/pastoreosistemas/04fisiologia_de_la_planta_pastoreada.htm. Accessed Sep 10, 2017.

26. Villegas AY, Hernández GA, Pérez PJ, López CC, Herrera HJG, Enríquez QJF, et al. Patrones estacionales de crecimiento de dos variedades de alfalfa (Medicago sativa L.). Téc Pecu Méx 2004;42(2):145-158.

27. Barbosa RA, Nacimiento-Jr D, Vilela HH, Da-Silva CS, Batista-Euclides PV, Sbrissia FA, Da-Lana SB. Morphogenic and structural characteristics of guinea grass pastures submitted to three frequencies and two defoliation severities. Rev Bras Zootec 2011;(40):947-954. 